\title{
Low genetic diversity of Plasmodium falciparum merozoite surface protein 1 and 2 and multiplicity of infections in western Ethiopia following effective malaria interventions
}

Geletta Tadele ( $\sim$ geletta98@yahoo.com )

Addis Ababa University

Fatou K.Jatieh

MRC Unit the Gambia

Mary Oboh

MRC Unit the Gambia

Eniyou Oriero

MRC Unit the Gambia

Sisay Dugassa

Addis Ababa University

Alfred Amambua-Ngwa

MRC Unit the Gambia

Lemu Golassa

Addis Ababa University

\section{Research Article}

Keywords: MOI, genetic diversity, P. falciparum, malaria transmission, western Ethiopia

Posted Date: February 24th, 2022

DOI: https://doi.org/10.21203/rs.3.rs-1385055/v1

License: (a) (i) This work is licensed under a Creative Commons Attribution 4.0 International License.

Read Full License 
Low genetic diversity of Plasmodium falciparum merozoite surface protein 1 and 2 and multiplicity of infections in western Ethiopia following effective malaria interventions

Geletta Tadele ${ }^{1}$,

Aklilu Lemma Institute of Pathobiology, Addis Ababa University, Addis Ababa, Ethiopia

Fatou K. Jaiteh ${ }^{2}$,

Medical research Council Unit the Gambia, London school of hygiene and Tropical Medicine, Gambia

Mary $\mathrm{Oboh}^{2}$,

Medical research Council Unit the Gambia at, London school of hygiene and Tropical Medicine,

Gambia

Eniyou Oriero $^{2}$,

Medical research Council Unit the Gambia at, London school of hygiene and Tropical Medicine, Gambia

Sisay Dugassa ${ }^{1}$,

Aklilu Lemma Institute of Pathobiology, Addis Ababa University, Addis Ababa, Ethiopia

Alfred Amambua-Ngwa ${ }^{2}$,

Medical research Council Unit the Gambia at, London school of hygiene and Tropical Medicine, Gambia

Lemu Golassa $^{1}$

1: Aklilu Lemma Institute of Pathobiology, Addis Ababa University, Addis Ababa, Ethiopia

Corresponding author:

GelettaTadele

geletta98@yahoo.com 
Background: Genetic diversity of malaria parasites can inform the intensity of transmission and poses a major threat to malaria control and elimination interventions. Characterization of the genetic diversity would provide essential information about the ongoing control efforts. This study aimed to explore allelic polymorphism of merozoite surface protein 1 ( $m s p l$ ) and merozoite surface protein 2 (msp2) to determine the genetic diversity and multiplicity of $P$. falciparum infections circulating in high and low transmission sites in western Ethiopia.

Method: Parasite genomic DNA was extracted from a total of 225 dried blood spot collected from confirmed uncomplicated $P$. falciparum malaria infected patients in western Ethiopia. Of these, $\underline{(163)} 72.4 \%$ and (62) $27.6 \%$ of the samples were collected in high and low transmission areas, respectively. Polymorphic $m s p 1$ and $m s p 2$ genes were used to explore the genetic diversity and multiplicity of falciparum malaria infections. Mspl genotyping was successful in 141/163 and 55/62 samples collected from high and low transmission areas, respectively. On the other hand, genotyping of msp 2 was carried out among $85.3 \%$ and $96.8 \%$ of the samples collected in high and low transmission sites, respectively. $P$. falciparum $m s p 1$ and $m s p 2$ genes were amplified by nested PCR and the PCR products were analyzed by Qiaxcel software. A p-value of less or equal to 0.05 was considered significant.

Result: High prevalence of falciparum malaria was identified in children less than 15 years as compared with those $\geq 15$ years old $(\mathrm{AOR}=2.438, \mathrm{P}=0.005)$. The three allelic families of $m s p 1$ (K1, MAD20 and RO33) and the two allelic families of msp2 (FC27 and 3D7), were observed in samples collected in high and low transmission areas. However, MAD 20 and FC 27 alleles were the predominant allelic families in both setting. $P$. falciparum isolates circulating in western Ethiopia had low genetic diversity and mean MOI. No difference in mean MOI between high transmission site (mean MOI 1.104) compared with low transmission area (mean MOI 1.08) (p> 0.05). Expected Heterozygosity of $m s p 1$ gene was slightly higher in isolates collected from high transmission site $(\mathrm{He}=0.17)$ than those isolates from low transmission $(\mathrm{He}=0.12)$. However, the heterozygosity of $m s p 2$ gene was not different in both settings (Pfmsp2: 0.04 in high transmission; pfmsp2: 0.03 in low transmission).

Conclusion: $P$. falciparum from clinical malaria cases in western Ethiopia have low genetic diversity and multiplicity of infection irrespective of the intensity of transmission at the site of sampling. These may be signalling the effectiveness of malaria control strategies in Ethiopia; 
although further studies are required to determine how specific intervention strategies and other parameters that drive the pattern.

Key words: MOI / genetic diversity/ P. falciparum/malaria transmission/ western Ethiopia

\section{Background}

Malaria remains a global public health problem with 229 million cases and 409000 deaths globally in 2019. Plasmodium falciparum remains the most dangerous malaria parasite species to humans with important public health consequences mostly in Africa (1).

Multiple Plasmodium strains (also known as multiple infections) is common in malaria-endemic areas, which may result from the bite of mosquitoes infected with more than one clone or from multiple bites (2). Interaction and competition of different strains for resources within a host during their life cycles may be important for survival and promotes diversity of the parasite (3, 4). The presence of multiple infecting strains may also affect key phenotypic traits, including drug resistance $(5,6)$ and risk of severe disease $(7,8)$. Characterizing genetic diversity of malaria in infected individuals and populations of different endemic settings could be helpful in determining the effectiveness of malaria interventions. Multiplicity of the parasite has been proposed as a marker to measure changes in transmission intensity in time and space $(9,10)$.

Malaria parasites genomic epidemiology studies have been assessed using multiplicity of infection (MOI) and the proportion of polyclonal infections (also termed 'multiple clone infections' $(9,11)$. It is generally assumed that MOI is higher in high transmission settings, while in low transmission settings, most individuals might carry single clone infections. A correlation between transmission intensity and MOI has been reported (12-14). But, a study done in Eswatini showed absence of association between multiplicity of infection and transmission intensity, most likely due to infections being imported (15). In Ethiopia, altitude, and climate (rainfall and temperature) are the most significant determinants for malaria transmission. $P$. falciparum is the dominant malaria parasite, accounting for 60 to $70 \%$ of all malaria cases (16).

Over the last two decades, malaria morbidity and mortality have been reduced dramatically in the country following intensive use of insecticide residual spray, long-lasting insecticide treated 
nets, chemotherapies, improved diagnosis and case management in recent years (17). Towards local strategies for malaria elimination; it is important to assess genetic diversity of malaria parasite in different settings to determine the effectiveness of the ongoing interventions and to better understand parasite population circulating in a given geographic area. In this study we focus on Western Ethiopia, where there is relatively limited information about the genetic diversity and MOI of $P$. falciparum infections. The aim of this study was to compare genetic diversity of $P$. falciparum isolates collected from high and low transmission sites, western Ethiopia.

\section{Methods}

\section{Study site and period}

This study was carried out during high malaria transmission season from September to December 2020 in four health centers located in areas with different levels of malaria transmission in western part of Ethiopia. These included Sherkole and Horazhab health centers which are found in Assosa zone in the Benishangul-Gumuz Region of Ethiopia. Both health centers are located in areas along the western borders with Sudan which has been characterized with the highest transmission intensity (18). Malaria transmission remains generally high in the western Ethiopian border area near Sudan (19). Sherkole health centre is located in Sherkole district which is bordered by Sudan in the north. Sherkole town is about $754 \mathrm{~km}$ from Addis Ababa. Horazhab health center is located in Kurmuk district, and it is bordered by Sudan in the north and west. It is about $769 \mathrm{~km}$ from Addis Ababa (figure 1).

Anger Gute and warabo health centers are found in Anger Gute town, Gida Ayana district, East Wollega Zone, Oromia Regional State. Anger Gute town is $360 \mathrm{Km}$ away from Addis Ababa. Malaria transmission in Anger Gute area is low and stable. P. falciparum infection prevalence among children 2-10 years was $<5 \%$ (18). Incidence of malaria in and around Anger Gute town was 3.43 per 1000 population at risk of the disease and the malaria trend between 2014 to 2018 indicated nearly unchanged numbers of malaria cases (20). 


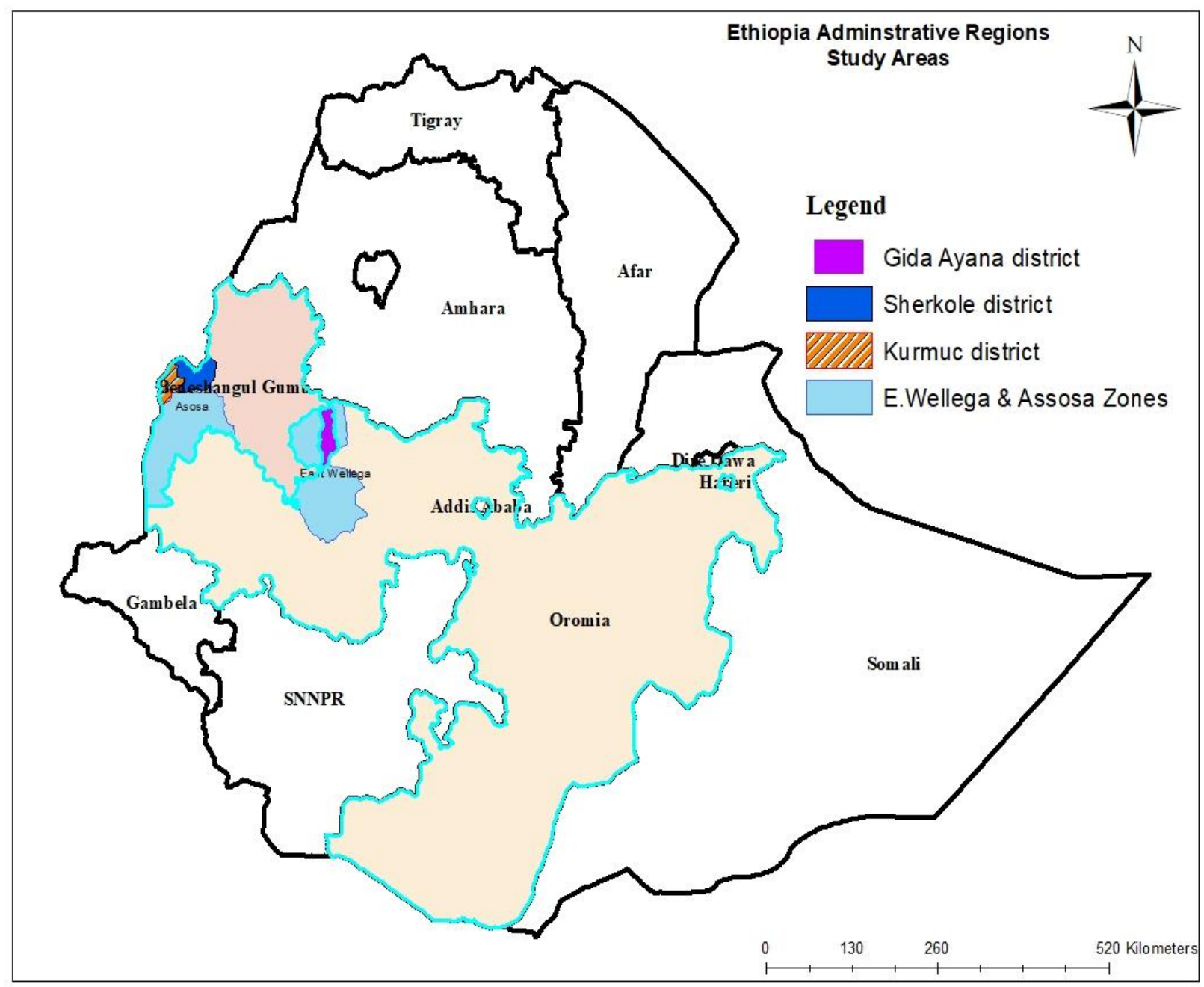

Figure 1. Map of Ethiopia showing the study area, Gida Ayana, Sherkole and Kurmuk districts, Western Ethiopia.

\section{Study design}

A health facility based cross-sectional study was conducted from September to December, 2020.

Consented febrile patients seeking malaria diagnosis at the study sites were recruited.

\section{Sample size}

The sample size was determined using a single population formula, using $13.1 \%$ prevalence of malaria in Benishangul-Gumuz regional state(16), a 95\% confidence level and 5\% precision. Accordingly, the calculated sample size was 175 . With $13 \%$ adjustment for non-response rate, a 
total of 198 uncomplicated falciparum malaria patients were included in the study. The number of patients included from high and low transmission sites were proportional to the number of confirmed $P$. falciparum reported from the study health facilities in 2019 G.C. Accordingly, from a total of 230 samples, 166 and 64 were collected from high and low transmission areas, respectively.

\section{Study population}

At each health center, consenting patients with uncomplicated malaria whose age was greater than 6 months, and microscopically-confirmed to be infected with P. falciparum, were enrolled. For confirmatory diagnosis of $P$. falciparum, finger-prick blood samples were used for thick and thin blood films for slide microscopy were made. Each slide was stained with $10 \%$ Giemsa for $10 \mathrm{~min}$ and 100 fields examined before designating a sample negative(21). Once the patients were microscopically confirmed of $P$. falciparum infection, they were consented to give finger-prick blood samples to prepare dried blood spots (DBS) on filter paper (Whatman No. 1001 320, International Ltd. Maidstone, England). The DBS were kept in plastic bags with desiccants until molecular analysis. Patients with mixed infections with other Plasmodium species were excluded from the study.

\section{Molecular genotyping}

Molecular detection of parasite genomic DNA was done at Medical Research Council Unit The Gambia at the London School of Hygiene \& Tropical Medicine. DNA extraction was done from DBS using the Chelex protocol as earlier described (22). P. falciparum detection was performed by Var gene acidic terminal sequence (varATS) real-time PCR as previously described (23). For genetic diversity, genomic DNA of the parasite was amplified by multiplex primary and Nested PCR using allele specific Primers ( Table 1) of $m s p 1$ and $m s p 2$ as per published protocol (24). Allelic variants of msp1 (K1, MAD 20 and RO33) and msp2 (FC27 and 3D7) were detected by allelic family-specific nested PCR amplification. Genotypes were distinguished using their fluorescent dye (indicating the allelic family) and by their size which was determined by Qiaxcel software. 


\section{Data analysis}

PCR amplicon bands for msp1 and msp2 were sized and binned using the Qiaxcel analysis software. SPSS 20.0 statistical software package (SPSS, Inc, Chicago, USA) was used for statistical analysis. The allelic frequency of $m s p 1$ or $m s p 2$ was determined by counting the number of samples observed with a particular allelic variant, divided by the total number of allelic variants observed in all the samples analyzed for $m s p 1$ or $m s p 2$.

An infection with a single allele for both targets was considered as a monoclonal infection and those infections with more than one PCR amplicon for either or both targets were considered as polyclonal. Mean MOI in both settings of malaria transmission were determined as the ratio of the total number of $P$. falciparum genotypes detected in $m s p l$ and $m s p 2$ by the number of samples positive for $m s p 1$ and $m s p 2$. Student's t test was used to compare the mean MOI of both loci between high and low transmission sites.

Expected heterozygosity (HE), a measure for genetic diversity, was calculated by using the following formula: $\mathrm{HE}=[\mathrm{n} /(\mathrm{n}-1)][(1-\Sigma$ pi2) $]$, where $\mathrm{n}$ is the number of isolates sampled and pi is the allele frequency at a given locus. A p-value of less or equal to 0.05 was considered suggestive of a statistically significant difference between sites.

\section{Ethical consideration}

Ethical clearance was obtained from the Ethiopian National Ethic Review Committee and Addis Ababa University, Aklilu Lemma Institute of Pathobiology, IRB. Permission to conduct the study at the health facilities was sought from the relevant regional and district health authorities. Written informed consent was obtained from adult study participants and a parent or guardian of a child. Written informed assent was also taken from children. 


\section{Results}

Out of 230 microscopically confirmed $P$. falciparum cases enrolled in the study, 225 of them tested positive by PCR. 72.4\% (163/225) and $27.6 \%$ (62/225) PCR positive samples were collected form high and low malaria transmission sites, respectively. The mean age of study participants was $17.8 \pm 12.7$ years and with an age range of 7 months -75 years old. Ratio of male to female was 1.27: 1 . The prevalence of falciparum malaria was higher in children less than 15 years as compared with those $\geq 15$ years old ( $\mathrm{AOR}=2.438, \mathrm{P}=0.005$ ). No significant difference in $P$. falciparum infections by gender was observed ( $\mathrm{p}>0.05)$, (Table 1).

Table 1: Sociodemographic characteristics and occurrence of malaria in western Ethiopia

\begin{tabular}{|ccccccc|}
\hline Variables & $\begin{array}{c}\text { High } \\
\text { transmission }\end{array}$ & $\begin{array}{c}\text { Low } \\
\text { transmission }\end{array}$ & COR & P-value & AOR & P-value \\
Sex Male & 85 & 41 & 1.79 & 0.061 & 1.827 & 0.057 \\
$\quad$ Female & 78 & 21 & 1 & & 1 & \\
Age categories & & & & & & \\
$<\mathbf{1 5}$ years & 90 & 21 & 2.41 & 0.005 & 2.438 & 0.005 \\
$\mathbf{2 1 5}$ years & 73 & 41 & 1 & & 1 & \\
\hline
\end{tabular}

\section{Genetic polymorphism of $m s p 1$ and $m s p 2$ genes}

Msp1 genotyping was successful in 87\% (141/163) and 88.7\% (55/62) of samples collected from high and low transmission areas, respectively. Similarly, for msp2 genotyping, $85.3 \%$ (139/163) and $96.8 \%$ (60/62) samples were successfully genotyped from high and low transmission sites, respectively. For $m s p 1$, the MAD20 allele was dominant, with a total of $87 \%, 25.5 \%$ and $5 \%$ of the samples from high transmission site had MAD20, K1 and RO33 allelic families of $m s p 1$, respectively. Similarly, 93\%, 14.5\% and 5\% samples collected from low malaria transmission setting had MAD20, K1 and RO33 msp1 allelic families, respectively. Multiclonal infections with K1+MAD20, MAD20+R033 combinations were also observed. In high transmission 
setting, K1+MAD20 accounted for $14.9 \%$ while the frequency of this allelic combination was $10.9 \%$ in low transmission setting (Figure 2). Allelic combinations of $\mathrm{K} 1$ and RO33 and K1/MAD20/RO33 were not detected in any infection.

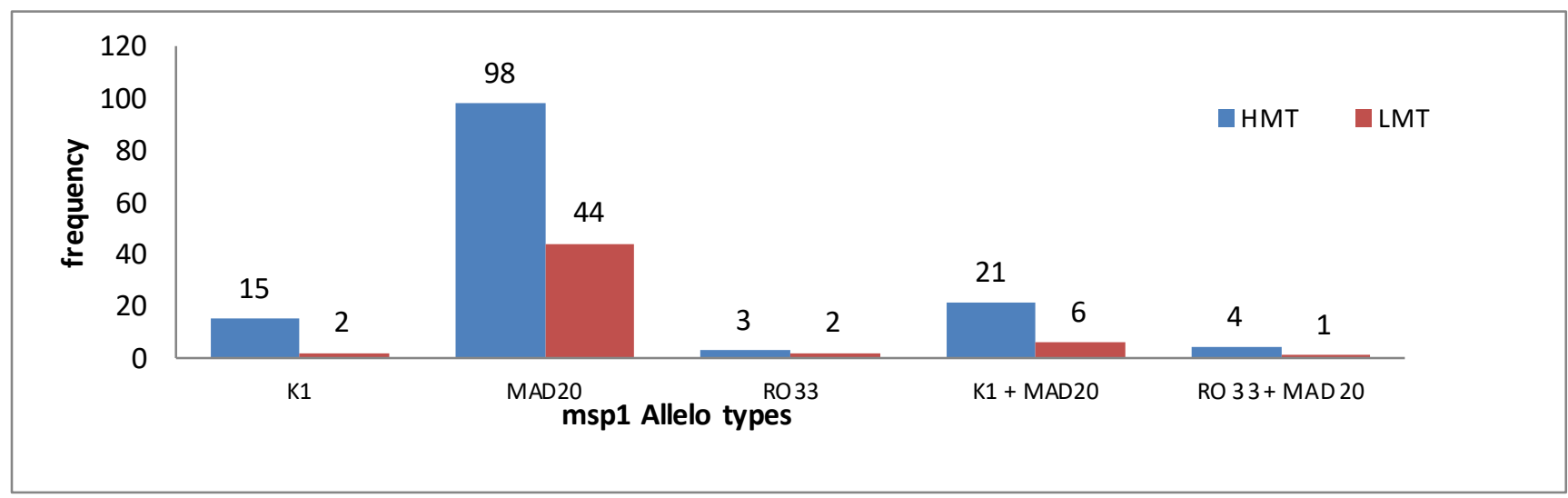

Figure 2: Distribution of $m s p-1$ allele types among P. falciparum isolates in different level of malaria transmission, western Ethiopia. HMT: high malaria transmission, LMT: low malaria transmission.

Both FC27 and 3D7 msp2 allelic types were identified among the samples collected from both study sites. However, FC27 was the frequent allelic type in western Ethiopia. There was no difference in the frequency of this allele in high transmission (94.96\%) and low transmission $(95 \%)$ areas. Few multi-allelic infections combining 3D7 +FC27 families were detected (Figure $3)$.

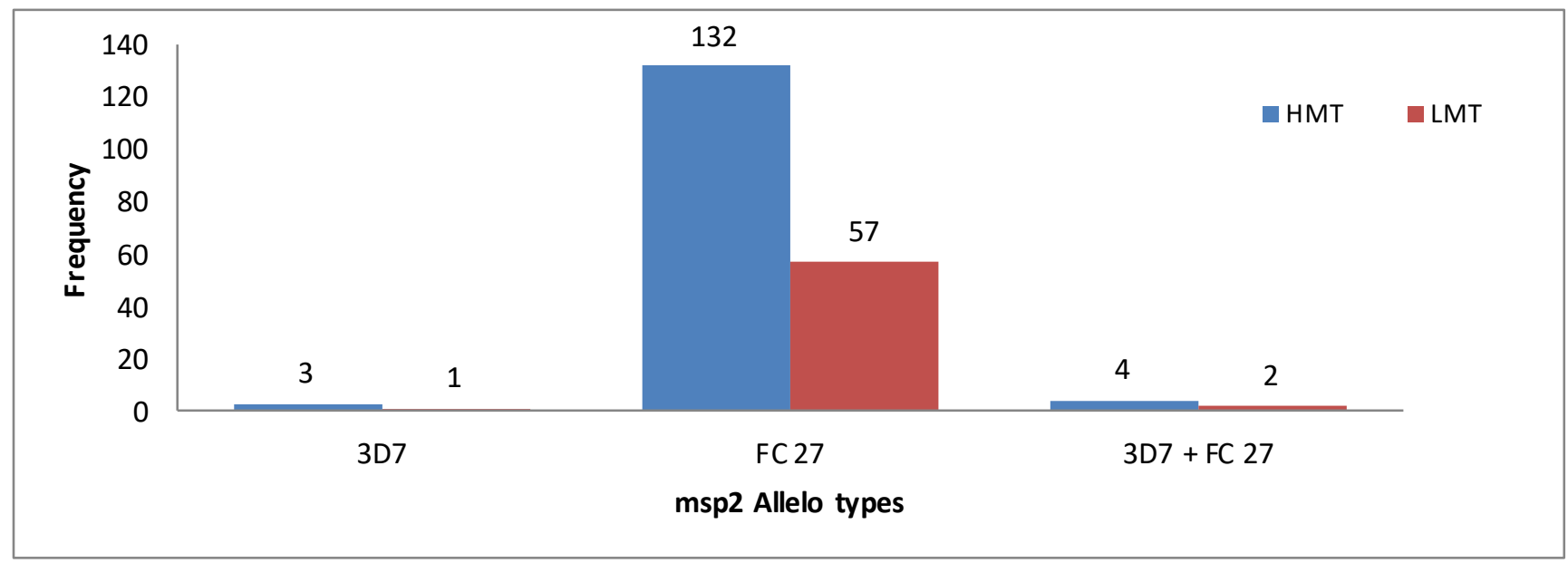

Figure 3: Distribution of $m s p 2$ Allelotypes by intensity of malaria transmission in western Ethiopia. 
In western Ethiopia, monoclonal $P$. falciparum infections were predominant, representing $82.3 \%(116 / 141)$ and $87.3 \%(48 / 55)$ of the samples genotyped for $m s p l$ in high and low transmission sites, respectively. Similarly, $97.2 \%$ (135/139) and 96.7 \% (58/60) of isolates from high and low transmission areas were monoclonal $m s p 2$, respectively. No significant difference was observed in the proportion of $m s p 1$ or $m s p 2$ multiplicity of infection with sex, age group of study participants and intensity of transmission $(\mathrm{P}>0.05)$ (Table 2).

Table 2: Proportion of multiplicity of $P$. falciparum infection in relation to sociodemographic indices of patients and level of malaria transmission, western Ethiopia

\begin{tabular}{|c|c|c|c|c|c|c|c|c|}
\hline \multirow[t]{2}{*}{ Variables } & \multicolumn{2}{|c|}{$\operatorname{msp1}(\mathrm{n}=196)$} & \multirow[t]{2}{*}{ AOR } & \multirow[t]{2}{*}{ P-value } & \multicolumn{2}{|c|}{$\operatorname{msp2}(\mathrm{n}=199)$} & \multirow[t]{2}{*}{ AOR } & \multirow[t]{2}{*}{ P-value } \\
\hline & monoclonal & polyclonal & & & monoclonal & polyclonal & & \\
\hline \multicolumn{9}{|l|}{ Sex } \\
\hline Male & 95 & 15 & 0.67 & 0.31 & 107 & 4 & 1.61 & 0.6 \\
\hline Female & 69 & 17 & & & 86 & 2 & & \\
\hline \multicolumn{9}{|l|}{ Age categories } \\
\hline$<15$ years & 82 & 15 & 1.2 & 0.64 & 134 & 3 & 0.95 & 0.931 \\
\hline$\geq 15$ years & 82 & 17 & 1 & & 58 & 3 & 1 & \\
\hline \multicolumn{9}{|c|}{ Level of transmission } \\
\hline High & 116 & 25 & 0.69 & 0.43 & 135 & 4 & 1.11 & 0.91 \\
\hline Low & 48 & 7 & 1 & & 58 & 2 & 1 & \\
\hline
\end{tabular}

P. falciparum isolates collected from western Ethiopia had low mean MOI and there was no significant difference in mean MOI between high transmission (mean MOI=1.104) and low transmission (mean MOI=1.08) ( $\mathrm{p}>0.05$ ). Expected Heterozygosity of mspl gene was slightly higher in high malaria intensity sites $(\mathrm{He}=0.17)$ than isolates from low malaria intensity $(\mathrm{He}=$ 0.12). On the other hand, heterozygosity of msp2 gene was not different in both settings (Pfmsp2: 0.04; pfmsp2: 0.03), (Table 3). 
Table 3: MOI and heterozygosity of $m s p 1$ and $m s p 2$ of $P$. falciparum in western Ethiopia

\begin{tabular}{|c|c|c|c|}
\hline Gene/genes & high transmission $(\mathrm{n}=\mathbf{2 8 0})$ & Low transmission $(n=115)$ & p- value \\
\hline mean MOI & 1.104 & 1.08 & 0.44 \\
\hline msp1 He & 0.17 & 0.12 & \\
\hline msp2 He & 0.04 & 0.03 & \\
\hline
\end{tabular}

\section{Discussion}

Measuring the complexity of infection and genetic diversity of parasite populations across different endemicities may be used as an indicator to evaluate the efficacy of ongoing control and elimination strategies. While a few studies have investigated MOI and genetic diversity of $P$. falciparum population circulating in western Ethiopia, these didn't represent populations relatively high and low transmission sites as presented here. The prevalence of uncomplicated falciparum malaria in the populations studied was higher in children $\leq 15$ years than those $\geq 15$ years old $(\mathrm{AOR}=2.438, \mathrm{P}=0.005)$ and this was in agreement with previous reports from Ethiopia and Kenya $(25,26)$. Immunity to malaria by an individual depends on their age, the number of infectious mosquito bites (27) and by the intensity of transmission(28, 29). In high transmission areas, burden of malaria is mainly among young children unlike in areas with low transmission, where the population has low exposure to infection and malaria occurs in all age groups $((25,30$, $31)$.

The three allelic families of $m s p 1(\mathrm{~K} 1, \mathrm{MAD} 20$ and RO33) and FC27 and 3D7 of $m s p 2$ allelic families were identified in the study areas. However, MAD 20 of $m s p 1$ and FC27 of $m s p 2$ allelic families were the predominant alleles in both areas with variable malaria endemicity. There was no difference in the frequency of $m s p l$ or $m s p 2$ allelic families by intensity of transmission. Therefore, population composition of $P$. falciparum isolates in western Ethiopia shows monoclonality infections regardless of the difference in intensity of transmission intensity and geographic separation of the parasites. This finding suggests the likely presence of high level of inbreeding among the circulating clones between the study sites. Previous reports had also indicated that malaria parasites in Ethiopia have moderate levels of genetic diversity and similar 
population structure of the parasite (32). They presented the lowest levels of heterozygosity in a continent-wide $P$. falciparum genomic analysis(33), indicating the need to further determine how they have evolved and are responding to the general interventions recommended for all malaria populations.

The predominance of the MAD 20 for $m s p 1$ and the FC27 allelic family for $m s p 2$ among $P$. falciparum isolates were inconsistent with previous studies in other parts of Africa; North Central Nigeria (34), Equatorial Guinea (35), and Northwest Ethiopia (36). Although this corroborates previous studies from Ethiopia finding similar patterns with the predominance of MAD 20 (37) and FC27 $(38,39)$, other reports had shown the predominance of K1 of msp1 and 3D7/IC1 of msp2 in southwestern Ethiopia (40). This is either due to variance between populations or changes that may have occurred over time following selection by pressure from interventions and reducing transmission intensity.

Most of the participants had monoclonal infections at both mspl or msp2 loci in both settings. As with previous reports, multiplicity of infection (MOI) was also not associated with age groups $(34,38)$. This was in contrast to other studies that found a correlation between polyclonal infection with age groups (41), though this was in a high transmission region in Burkina-Faso in west Africa. MOI was not associated with sex of the patients as report from Burkina Faso (42). Overall, P. falciparum isolates from western Ethiopia showed low mean MOI and limited genetic diversity, with no differences based on intensity of transmission, a pattern that has been seen in some malaria endemic regions of Africa, the prevalence of infections have generally become low and imported cases are common $(15,43,44)$. Examples of these include Senegal and Eswatini, which are now heading towards pre-elimination. Though high MOI, and genetic diversity has been associated with higher malaria-endemicity, these indices as determined by mspl and msp2 typing may not be sensitive enough at a lower overall transmission level as seen in Ethiopia $(12,35,45,46)$. However, the lack of differences with the relative variance in transmission intensities might be an indication that malaria control measures that reduce entomological inoculation rate $(47,48)$, and deployment of artemisinin-combination therapy that reduce population diversity by removing the drug sensitive parasites(49) have been effective in Ethiopia. It is also possible differences in human demography, ecology and Anopheles mosquito vector might be shaping $P$. falciparum population structure in malaria endemic sites (50-52). 
Within-host competitive interactions of $P$. falciparum may also determine diversity of the parasite (3). Malaria transmission in Ethiopia is driven mainly by Anopheles arabiensis while An. pharoensis, An. funestus and An. nili are secondary vectors(53, 54), unlike the mostly An gambiae seen across the most endemic regions of west and central Africa.

Conclusion: P. falciparum isolates were mainly monoclonal with low MOI and lesser genetic diversity. Mean MOI and diversity of the parasite were not associated with intensity of malaria transmission. Hence, MOI and genetic diversity of $P$. falciparum might not be a good predictor of transmission intensity in all malaria endemic areas. There is a need to find out an inclusive measurement of intensity of transmission in all malaria endemic areas. The observation of low genetic diversity and multiplicity of infections in the study area may signal the effectiveness of the recent deployment of massive malaria control strategies in Ethiopia; however, this needs further studies to map the different intervention strategies and other parameters that could affect parasite genetic diversity.

Abbreviations: DBS: dried blood spots; IRB: Institute Review Board; PCR: polymerase chain reaction; varATS: Var gene acidic terminal sequence; MOI: multiplicity of infection; mspl: merozoite surface protein $1 ;$ msp2: merozoite surface.

\section{Acknowledgements}

The authors would like to thank all $P$. falciparum patients for their willingness to participate in the study. We would also like to thank Medical Research Council Unit the Gambia, at London school of hygiene and Tropical Medicine, Gambia for the permission to use the molecular biology laboratory and the PAMGEN H3Africa project for financial support.

\section{Authors' contributions}

Conceptualization: Dr. Lemu Golassa and Geletta Tadele

Supervision of the overall work: Dr. Lemu Golassa, Dr. Alfred Amambua-Ngwa and Dr. Sisay Dugassa

Methodology: Geletta Tadele and Dr. Lemu Golassa

Laboratory work: Geletta Tadele, Fatou K. Jaiteh, Eniyou Oriero and Mary Oboh 
write up of the original draft of the manuscript: Geletta Tadele

Reviewing and Editing the manuscript: Dr. Lemu Golassa, Dr. Alfred Amambua-Ngwa, Dr. Sisay Dugassa, Eniyou Oriero and Mary Oboh

All authors read and approved the final manuscript.

\section{Funding}

This work was supported by the PAMGEN H3 Africa project (H3A/002/18) and Addis Ababa University, Post Graduate studies.

\section{Availability of data and materials}

The datasets and analyzed result of the study are available on the corresponding author and can be obtained on reasonable request.

\section{Ethics approval and consent to participate}

Ethical approval was obtained from the Ethiopian National Ethic review committee, Addis Ababa, Ethiopia (No. MoSHE 04/246/66) and Aklilu Lemma Institute of Pathobiology, Institutional review board, Addis Ababa University (No. ALIPB IRB/ 19/2012/20). Written informed consent was obtained from adult study participants and a parent or guardian of a child. Written informed assent was also taken from children.

\section{Consent for publication}

Not applicable.

\section{Competing interests}

The authors declare that they have no competing interests.

\section{Author details}

1. Aklilu Lemma Institute of Pathobiology, Addis Ababa University, Addis Ababa, Ethiopia

2. Medical research Council Unit the Gambia at, London school of hygiene and Tropical Medicine, Banjul, Gambia 
References

1. WHO(2010). Global report on antimalarial drug efficacy and drug resistance: 2000-2010.

2. Nkhoma SC, Trevino SG, Gorena KM, Nair S, Khoswe S, Jett C, et al(2020). Co-transmission of related malaria parasite lineages shapes within-host parasite diversity. Cell host \& microbe;27(1):93-103. e4.

3. Bashey F. Within-host competitive interactions as a mechanism for the maintenance of parasite diversity(2015). Philosophical Transactions of the Royal Society B: Biological Sciences;370(1675):20140301.

4. He Q, Pilosof S, Tiedje KE, Day KP, Pascual M(2021). Frequency-Dependent competition between strains imparts persistence to perturbations in a model of Plasmodium falciparum malaria transmission. frontiers in Ecology and Evolution:doi.org/10.3389/fevo.2021.633263

5. Bushman M, Morton L, Duah N, Quashie N, Abuaku B, Koram KA, et al(2018). Within-host competition and drug resistance in the human malaria parasite Plasmodium falciparum. Proceedings of the Royal Society B: Biological Sciences;283(1826):20153038.

6. Kyabayinze DJ, Karamagi C, Kiggundu M, Kamya MR, Wabwire-Mangen F, Kironde F, et al(2008). Multiplicity of Plasmodium falciparum infection predicts antimalarial treatment outcome in Ugandan children. African health sciences;8(4):200-5.

7. Sondo P, Derra K, Lefevre T, Diallo-Nakanabo S, Tarnagda Z, Zampa O, et al(2019). Genetically diverse Plasmodium falciparum infections, within-host competition and symptomatic malaria in humans. Scientific reports;9(1):1-9.

8. Kiwuwa MS, Ribacke U, Moll K, Byarugaba J, Lundblom K, FÃårnert A, et al(2013). Genetic diversity of Plasmodium falciparum infections in mild and severe malaria of children from Kampala, Uganda. Parasitology research;112(4):1691-700.

9. Tusting LS, Bousema T, Smith DL, Drakeley C(2014). Measuring changes in Plasmodium falciparum transmission: precision, accuracy and costs of metrics. Advances in parasitology;84:151-208.

10. Lopez L, Koepfli C(2021). Systematic review of Plasmodium falciparum and Plasmodium vivax polyclonal infections: Impact of prevalence, study population characteristics, and laboratory procedures. Plos one;16(6):e0249382.

11. Zhong D, Koepfli C, Cui L, Yan G(2018). Molecular approaches to determine the multiplicity of Plasmodium infections. Malaria journal;17(1):1-9.

12. Fola AA, Harrison GA, Hazairin MH, Barnadas Cl, Hetzel MW, Iga J, et al(2017). Higher complexity of infection and genetic diversity of Plasmodium vivax than Plasmodium falciparum across all malaria transmission zones of Papua New Guinea. The American journal of tropical medicine and hygiene;96(3):630.

13. Funwei RI, Thomas BN, Falade CO, Ojurongbe O(2018). Extensive diversity in the allelic frequency of Plasmodium falciparum merozoite surface proteins and glutamate-rich protein in rural and urban settings of southwestern Nigeria. Malaria journal;17(1):1-8.

14. Touray AO, Mobegi VA, Wamunyokoli F, Herren JK(2020). Diversity and Multiplicity of $P$. falciparum infections among asymptomatic school children in Mbita, Western Kenya. Scientific reports;10(1):1-8.

15. Roh ME, Tessema SK, Murphy M, Nhlabathi N, Mkhonta N, Vilakati S, et al(2019). High genetic diversity of Plasmodium falciparum in the low-transmission setting of the Kingdom of Eswatini. The Journal of infectious diseases;220(8):1346-54.

16. Assefa A(2015).The third Ethiopian Malaria Indicator Survey 2015 (EMIS-2015). 28th 
Annual conference, 2016.

17. Bugssa G, Tedla K. Feasibility of Malaria Elimination in Ethiopia. Ethiopian Journal

of Health Sciences;30(4): 607-614.

18. Hiwot Solomon Taffese, Henok Kebede Ejigu, Meshesha Balkew ,Bernard Mitto, Punam Amratia, Judy Omumbo, Abdisalan M Noor \& Robert W Snow ( 2014). An Epidemiological Profile of Malaria in Ethiopia.

19. Taffese HS, Hemming-Schroeder E, Koepfli C, Tesfaye G, Lee M-c, Kazura J, et al(2018). Malaria epidemiology and interventions in Ethiopia from 2001 to 2016. Infectious diseases of poverty; $7(06): 1-9$.

20. Dufera M, Kenea O, Tadele G(2020). Malaria Incidence and Associated Risk Factors in and Around Anger Gute Town, Western Ethiopia. DOI: https://doi.org/10.21203/rs.3.rs-29458/v1

21. WHO(2010). Basic malaria microscopy: Part I. Learner's guide: World Health Organization.

22. Simon N, Shallat J, Williams Wietzikoski C, Harrington WE(2020). Optimization of Chelex 100 resin-based extraction of genomic DNA from dried blood spots. Biology Methods and Protocols;5(1):bpaa009.

23. Hofmann N, Mwingira F, Shekalaghe S, Robinson LJ, Mueller I, Felger I(2015). Ultrasensitive detection of Plasmodium falciparum by amplification of multi-copy subtelomeric targets. PLoS medicine;12(3):e1001788.

24. Foley M, Ranford-Cartwright LC, Babiker HA(1992). Rapid and simple method for isolating malaria DNA from fingerprick samples of blood. Molecular and biochemical parasitology;53(1-2):241-4.

25. Kamau A, Mtanje G, Mataza C, Mwambingu G, Mturi N, Mohammed S, et al(2020). Malaria infection, disease and mortality among children and adults on the coast of Kenya. Malaria journal;19(1):1-12.

26. Tegegne Y, Worede A, Derso A, Ambachew S(2021). The Prevalence of Malaria among Children in Ethiopia:A Systematic Review and Meta-Analysis. Journal of Parasitology Research; doi: 10.1155/2021/6697294

27. White M, Watson J(2018). Malaria: age, exposure and immunity. Elife;7:e40150.

28. Trape J-Fo, Rogier C(1996). Combating malaria morbidity and mortality by reducing transmission. Parasitology today;12(6):236-40.

29. Coulibaly D, Guindo B, Niangaly A, Maiga Fa, Konate S, Kodio A, et al(2021 ). A decline and age shift in malaria incidence in rural Mali following implementation of seasonal malaria chemoprevention and indoor residual spraying. The American Journal of Tropical Medicine and Hygiene; 104(4):1342.

30. Carneiro I, Roca-Feltrer A, Griffin JT, Smith L, Tanner M, Schellenberg JA, et al(2010). Age-patterns of malaria vary with severity, transmission intensity and seasonality in subSaharan Africa: a systematic review and pooled analysis. PloS one;5(2):e8988.

31. Kleinschmidt I, Sharp B(2001). Patterns in age and• specific malaria incidence in a population exposed to low levels of malaria transmission intensity. Tropical medicine \& international health;6(12):986-91.

32. Lo E, Hemming-Schroeder E, Yewhalaw D, Nguyen J, Kebede E, Zemene E, et al(2017). Transmission dynamics of co-endemic Plasmodium vivax and P. falciparum in Ethiopia and prevalence ofantimalarial resistant genotypes. PLoS neglected tropical diseases;11(7):e0005806.

33. Amambua-Ngwa A, Amenga-Etego L, Kamau E, Amato R, Ghansah A, Golassa L, et 
al(2019). Major

subpopulations of Plasmodium falciparum in sub-Saharan Africa. Science;365(6455):813-6.

34. Oyedeji SI, Bassi PU, Oyedeji SA, Ojurongbe O, Awobode HO(2020). Genetic diversity and complexity of Plasmodium falciparum infections in the microenvironment among siblings of the same household in North-Central Nigeria. Malaria Journal;19(1):1-10.

35. Chen J-T, Li J, Zha G-C, Huang G, Huang Z-X, Xie D-D, et al(2018). Genetic diversity and allele frequencies of Plasmodium falciparum msp1 and msp2 in parasite isolates from Bioko Island, Equatorial Guinea.Malaria journal;17(1):1-9.

36. Mohammed H, Kassa M, Mekete K, Assefa A, Taye G, Commons RJ(2018). Genetic diversity of the msp-1, msp-2, and glurp genes of Plasmodium falciparum isolates in Northwest Ethiopia. Malaria journal;17(1):1-8.

37. File T, Chekol T, Solomon G, Dinka H, Golassa L(2021). Detection of High Frequency of MAD20 Allelic Variants of Plasmodium Falciparum Merozoite Surface Protein 1 Gene from Adama and its Surroundings, Oromia, Ethiopia.Malaria journal; 20(385):1-10.

38. Mohammed H, Hassen K, Assefa A, Mekete K, Tadesse G, Taye G, et al(2019). Genetic diversity of Plasmodium falciparum isolates from patients with uncomplicated and severe malaria based on msp-1 and msp-2 genes in Gublak, North West Ethiopia. Malaria journal;18(1):1-8.

39. Abamecha A, El-Abid H, Yilma D, Addisu W, Ibenthal A, Bayih AG, et al(2020). Genetic diversity and genotype multiplicity of Plasmodium falciparum infection in patients with uncomplicated malaria in Chewaka district, Ethiopia. Malaria journal;19:1-9.

40. Mohammed H, Mindaye T, Belayneh M, Kassa M, Assefa A, Tadesse M, et al(2015). Genetic diversity of Plasmodium falciparum isolates based on MSP-1 and MSP-2 genes from Kolla-Shele area, Arbaminch Zuria District, southwest Ethiopia. Malaria journal;14(1):1-7.

41. Sondo P, Derra K, Rouamba T, Diallo SN, Taconet P, Kazienga A, et al(2020). Determinants of Plasmodium falciparum multiplicity of infection and genetic diversity in Burkina Faso. Parasites \&vectors;13(1):1-12.

42. Sondo P, Derra K, Rouamba T, Nakanabo Diallo S, Taconet P, Kazienga A, et al(2020). Determinants of Plasmodium falciparum multiplicity of infection and genetic diversity in Burkina Faso. Parasites \& vectors;13(1):1-12.

43. Ralinoro F, Rakotomanga TA, Rakotosaona R, Rakoto DAD, Menard D, Jeannoda V, et al(2021). Genetic diversity of Plasmodium falciparum populations in three malaria transmission settings in Madagascar. Malaria Journal;20(1):1-11.

44. Niang M, Loucoubar C, Sow A, Diagne MM, Faye O, Faye O, et al(2016). Genetic diversity of Plasmodium falciparum isolates from concurrent malaria and arbovirus co-infections in Kedougou, southeastern Senegal. Malaria journal;15(1):1-8.

45. Oboh MA, Ndiaye T, Diongue K, Ndiaye YD, Sy M, Deme AB, et al(2021). Allelic diversity of MSP1 and MSP2 repeat loci correlate with levels of malaria endemicity in Senegal and Nigerian populations. alaria journal;20(1):1-8.

46. Kateera F, Nsobya SL, Tukwasibwe S, Mens PF, Hakizimana E, Grobusch MP, et al(2016). Malaria case clinical profiles and Plasmodium falciparum parasite genetic diversity: a cross sectional survey at two sites of different malaria transmission intensities in Rwanda. Malaria journal;15(1):1-10.

47. Chitnis N, Hardy D, Gnaegi G, Boutsika K, Maire N, Steketee R, et al(2010). Modeling the effects of vector control interventions in reducing malaria transmission, morbidity and mortality. Malaria Journal;9(2):1-2. 
48. Shaukat AM, Breman JG, McKenzie FE(2010). Using the entomological inoculation rate to assess the impact of vector control on malaria parasite transmission and elimination. Malaria journal;9(1):1-12.

49. Ndiaye YD, Hartl DL, McGregor D, Badiane A, Fall FB, Daniels RF, et al(2021). Genetic surveillance for monitoring the impact of drug use on Plasmodium falciparum populations. International Journal for Parasitology: Drugs and Drug Resistance;17:12-22.

50. Annan Z, Durand P, Ayala FJ, Arnathau Cl, Awono-Ambene P, Simard Fdr, et al(2007). Population genetic structure of Plasmodium falciparum in the two main African vectors, Anopheles gambiae and Anopheles funestus. Proceedings of the National Academy of Sciences;104(19):7987-92.

51. Morlais I, Nsango SE, Toussile W, Abate L, Annan Z, Tchioffo MT, et al(2015). Plasmodium falciparum mating patterns and mosquito infectivity of natural isolates of gametocytes. PloS one;10(4):e0123777.

52. Tagliamonte MS, Yowell CA, Elbadry MA, Boncy J, Raccurt CP, Okech BA, et al(2020). Genetic Markers of Adaptation of Plasmodium falciparum to Transmission by American Vectors Identified in the Genomes of Parasites from Haiti and South America. Msphere;5(5):e00937-20.

53. $\mathrm{MOH}($ 2012). National malaria guidelines.

54. The President's Malaria Initiative (2017). Eleventh Annual Report to Congress. 